PROCEEDINGS OF THE

AMERICAN MATHEMATICAL SOCIETY

Volume 140, Number 7, July 2012, Pages 2267-2277

S 0002-9939(2011)11147-X

Article electronically published on November 1, 2011

\title{
THE EXCEPTIONAL SET IN VOJTA'S CONJECTURE FOR ALGEBRAIC POINTS OF BOUNDED DEGREE
}

\author{
AARON LEVIN
}

(Communicated by Matthew A. Papanikolas)

\begin{abstract}
We study the dependence on various parameters of the exceptional set in Vojta's conjecture. In particular, by making use of certain elliptic surfaces, we answer in the negative the often-raised question of whether Vojta's conjecture holds when extended to all algebraic points (that is, if the conjecture holds without fixing a bound on the degree of the algebraic points).
\end{abstract}

\section{INTRODUCTION}

Inspired by results in Nevanlinna theory, Vojta 22, 24, made the following deep conjecture in Diophantine approximation for algebraic points of bounded degree in a nonsingular complete variety.

Conjecture 1.1 (Vojta's Conjecture). Let $X$ be a nonsingular complete variety with canonical divisor $K$. Let $D$ be a normal crossings divisor on $X$, and let $k$ be a number field over which $X$ and $D$ are defined. Let $S$ be a finite set of places of $k, A$ a big divisor on $X, \epsilon>0$, and $r$ a positive integer. Then there exists a proper Zariski-closed subset $Z=Z(r, \epsilon, X, D, A, k, S)$ of $X$ such that

$$
m_{D, S}(P)+h_{K}(P) \leq d(P)+\epsilon h_{A}(P)+O(1)
$$

for all points $P \in X(\bar{k}) \backslash Z$ with $[k(P): k] \leq r$.

Vojta's Conjecture relates several fundamental arithmetic quantities associated to an algebraic point on a nonsingular complete variety. In the next section we will recall the definitions of several of the terms in the conjecture. Briefly, $m_{D, S}$ is a proximity function, measuring the part of the height relative to $D$ coming from places lying above places in $S ; h_{K}$ and $h_{A}$ are absolute logarithmic height functions associated to the divisors $K$ and $A$, respectively; and $d(P)=\frac{1}{[k(P): \mathbb{Q}]} \log \left|D_{k(P) / \mathbb{Q}}\right|$ is the absolute logarithmic discriminant. The set $Z$ is called the exceptional set. It will be our primary object of study here.

Vojta's Conjecture has a wide range of important consequences (see 22]), of which we just mention the $a b c$ Conjecture and the Bombieri-Lang Conjecture on rational points on varieties of general type. Various strengthened versions of Vojta's Conjecture have frequently appeared in the literature, often asserting that the

Received by the editors November 2, 2010 and, in revised form, February 20, 2011.

2010 Mathematics Subject Classification. Primary 11J97; Secondary 11J25.

This research was partially supported by NSF grant DMS-0635607.

(C)2011 American Mathematical Society Reverts to public domain 28 years from publication 
exceptional set $Z$ in the conjecture can be chosen independently of some of the parameters appearing in the conjecture. It is this aspect of Vojta's Conjecture that we wish to study here, that is, the dependence of $Z$ on the parameters $r, \epsilon, X, D, A, k$, and $S$.

That the exceptional set $Z$ must depend on $X$ and $D$ is trivial (indeed, $m_{D, S}(P)$ is not even well-defined for points in the support of $D$ ). The first nontrivial general observation regarding the dependence of $Z$ on the parameters is due to Vojta himself, who showed in 23 that, in general, the set $Z$ must be allowed to depend on the parameter $\epsilon$. Vojta's example in [23] used quadratic points (i.e., $r=2$ ). In [11, it was shown that $Z$ must be allowed to depend on $\epsilon$ even when the conjecture is restricted to rational points $(r=1)$. Of course, in specific cases, it may happen that $Z$ can be chosen independently of $\epsilon$. For instance, in Schmidt's Subspace Theorem $\left(r=1, X=\mathbb{P}^{n}, D\right.$ a sum of hyperplanes in general position), one of the few cases where Vojta's Conjecture is known, Vojta showed [23] that the exceptional set $Z$ (which can be taken to consist only of hyperplanes) can in fact be taken to be independent of the parameter $\epsilon$. We note also that the dependence of $Z$ on $\epsilon$ has ramifications for the form of the error term in (1.1). In particular, contrary to a conjecture of Lang 8 for the error term in Roth's theorem $\left(X=\mathbb{P}^{1}\right.$, $r=1$ ), in higher dimensions one cannot replace the term $\epsilon h_{A}(P)$ in (1.1) by a term like $\log h_{A}(P)$. In fact, if one considers Vojta's Conjecture with "truncated counting functions", by a result of Stewart and Tijdeman [19] (see also [20]), this same phenomenon occurs even in the one-dimensional case, where the error term is essentially the same as the error term in the $a b c$ Conjecture (see 21]).

In Section 3 we show that $Z$ must be allowed to depend on the big divisor $A$. In allowing arbitrary big divisors $A$, this is hardly surprising since the associated height $h_{A}$ may not even be bounded from below for points in the base locus of the linear system $|A|$. However, we will show that even if one restricts to ample divisors $A$ and rational points $(r=1)$, the set $Z$ must be allowed to depend on $A$. Since for any two ample divisors $A$ and $A^{\prime}$ on a variety $X$ we have $h_{A} \gg \ll h_{A^{\prime}}$, this phenomenon is necessarily closely related to the dependence of $Z$ on $\epsilon$.

We have nothing new to offer regarding the dependence of $Z$ on the number field $k$ or the set of places $S$. If $Z$ could not be taken independent of $k$ and $S$, this would imply that the exceptional set $Z$ is of a highly nongeometric nature, violating the spirit of Vojta's Conjecture. Consequently, Conjecture 1.1 is frequently stated with the additional hypothesis that $Z$ can be chosen independently of $k$ and $S$. Adopting this point of view, from now on we will notationally omit the (possible) dependence of $Z$ on $k$ and $S$.

Finally, we will give examples showing that the exceptional set $Z$ in Vojta's Conjecture must be allowed to depend on the parameter $r$. Explicitly, we will show this for a large class of elliptic surfaces.

Theorem 1.2. Let $X$ be a semistable elliptic surface over $\mathbb{P}^{1}$ with a section over a number field $k$. Let $D$ be a sum of fibers of $X$ containing the singular fibers. If Vojta's Conjecture holds for $X$ and $D$, then the exceptional set $Z(r, \epsilon, X, D, A)$ must depend on the parameter $r$.

We can also produce similar examples (with a different, but related, class of surfaces $X$ ) where $D=0$ (Theorem 4.7).

The rough idea of the construction behind Theorem 1.2 is as follows. We begin with a semistable elliptic surface $X$ over $\mathbb{P}^{1}$ with zero section $\sigma$, everything defined 
over some number field $k$. Let $D$ be the sum of the singular fibers. We construct a set of integral points $T$ on $\sigma \backslash D$ of some bounded degree (i.e., $[k(P): k]$ is bounded for $P \in T)$. For such points $P$ in $T$ we have $m_{D, S}(P)=h_{D}(P)$ and so the left-hand side of Vojta's Conjecture will be large. In fact, it will be large enough to force $\sigma$ to be in the exceptional set (for an appropriate choice of $r$ ). Now we use the fiberwise multiplication-by- $m$ map $[m]$ on the elliptic surface to pull back the points in $T$ to obtain certain points on torsion multisections. Infinitely many of these points will again violate inequality (1.1) of Vojta's Conjecture. The basic intuitive reason behind this is that Vojta's Conjecture and the terms in Vojta's Conjecture are well-behaved with respect to unramified maps. We take advantage of a logarithmic version of this, using the facts that the points in $T$ are integral with respect to $D$ and $[m]$ is unramified on $X \backslash D$. We conclude that, for large enough $r,[m]^{-1}(\sigma \backslash D)$ must be contained in the exceptional set in Vojta's Conjecture. Now varying $m$ gives the desired result.

As a consequence of Theorem 1.2, we obtain that Vojta's Conjecture does not hold uniformly for all algebraic points, answering a question that has frequently been raised (e.g., 22, p. 64] or [9, p. 223]). More precisely, in Section 4] we will show:

Theorem 1.3. Vojta's Conjecture does not hold when extended to $r=\infty$ (i.e., to all algebraic points), even when allowing the $O(1)$ term to depend on $[k(P): k]$. Specifically, there exists a nonsingular projective surface $X$, defined over a number field $k$, with canonical divisor $K_{X}$ and big divisor $A$, such that for any $\delta>0$, any constants $c_{i} \in \mathbb{R}, i \in \mathbb{N}$, and any sufficiently small $\epsilon>0$, the inequality

$$
h_{K_{X}}(P)>\left(\frac{3}{2}-\delta\right) d(P)+\epsilon h_{A}(P)+c_{[k(P): k]}
$$

holds for a Zariski-dense set of points $P$ in $X(\bar{k})$.

This is somewhat surprising since the analogous function field version of Vojta's Conjecture has been proved for curves (independently by McQuillan [13. and Yamanoi [25]) with $r=\infty$. Moreover, previous results in the equidimensional Nevanlinna theory of coverings of $\mathbb{C}^{n}[3$, , [10, Ch. IV] also appeared to support the possibility that Vojta's Conjecture would hold uniformly for all algebraic points.

Despite Theorem 1.3. it is still quite plausible that Vojta's Conjecture may hold uniformly for all algebraic points after making some simple modifications to the terms in the inequality (1.1). One possibility is given by the original form of the conjecture proposed in 22. In 22, Vojta included a $\operatorname{dim} X$ factor in the conjecture in front of the discriminant term $d(P)$, but this factor was later thought to be unnecessary (see, e.g., the comment after Conjecture 2.1 in [24]). None of the examples presented here prevent the possibility that Conjecture 1.1 could hold for all algebraic points with a $(\operatorname{dim} X) d(P)$ term in the inequality (1.1) (instead of $d(P))$. More precisely, our examples allow the possibility that Conjecture 1.1 could hold for all algebraic points when the $d(P)$ term is replaced by $\frac{3}{2} d(P)$ in the inequality (1.1). At the end of Section 4 we will also discuss some recent examples 1] of Autissier, Chambert-Loir, and Gasbarri concerning a geometric analogue of (1.1), which may provide further insight into the correct form of a uniform version of Vojta's Conjecture. 


\section{NotATION AND DEFINITIONS}

Throughout, $k$ will denote a number field, $\mathcal{O}_{k}$ the ring of integers of $k$, and $\mathcal{O}_{k}^{*}$ the group of units of $\mathcal{O}_{k}$. Associated to $k$ we have a canonical set $M_{k}$ of places (or absolute values) of $k$ consisting of one place for each prime ideal $\mathfrak{p}$ of $\mathcal{O}_{k}$, one place for each real embedding $\sigma: k \rightarrow \mathbb{R}$, and one place for each pair of conjugate embeddings $\sigma, \bar{\sigma}: k \rightarrow \mathbb{C}$. Let $k_{v}$ denote the completion of $k$ with respect to $v$. We normalize our absolute values so that for a prime $p \in \mathbb{Z},|p|_{v}=p^{-\left[k_{v}: \mathbb{Q}_{p}\right] /[k: \mathbb{Q}]}$ if $v$ corresponds to $\mathfrak{p}$ and $\mathfrak{p} \mid p$, and $|x|_{v}=|\sigma(x)|^{\left[k_{v}: \mathbb{R}\right] /[k: \mathbb{Q}]}$ if $v$ corresponds to an embedding $\sigma$ (in which case we say that $v$ is Archimedean). If $v$ is a place of $k$ and $w$ is a place of a field extension $L$ of $k$, then we say that $w$ lies above $v$, or $w \mid v$, if $w$ and $v$ define the same topology on $k$.

Let $D$ be a divisor on a nonsingular complete variety $X$, with both $X$ and $D$ defined over a number field $k$. Let $v \in M_{k}$. Extend $|\cdot|_{v}$ to an absolute value on $\bar{k}_{v}$. Let Supp $D$ denote the support of $D$. We define a local Weil function (or local height function) for $D$ relative to $v$ to be a function $\lambda_{D, v}: X\left(\bar{k}_{v}\right) \backslash \operatorname{Supp} D \rightarrow \mathbb{R}$ such that if $D$ is represented locally by $(f)$ on an open set $U$, then

$$
\lambda_{D, v}(P)=-\log |f(P)|_{v}+\alpha_{v}(P),
$$

where $\alpha_{v}$ is a continuous function on $U\left(\bar{k}_{v}\right)$ in the $v$-topology. Any two choices of $\lambda_{D, v}$ differ only by a bounded function on $X\left(\bar{k}_{v}\right) \backslash \operatorname{Supp} D$. Viewing $\bar{k} \subset \bar{k}_{v}$, we may also view $\lambda_{D, v}$ as a function on $X(\bar{k}) \backslash \operatorname{Supp} D$. The functions $\lambda_{D, v}$ give a measure of the $v$-adic distance of a point $P$ from $D$ (being large when $P$ is $v$-adically close to $D$ ). If $S$ is a finite set of places of $k$, we define the proximity function $m_{D, S}(P)$ (relative to $D$ and $S$ ) for $P \in X(\bar{k}) \backslash \operatorname{Supp} D$ by

$$
m_{D, S}(P)=\sum_{v \in S} \sum_{\substack{w \in M_{k(P)} \\ w \mid v}} \lambda_{D, w}(P) .
$$

Associated to $D$ we also have an absolute logarithmic height function on $X(\bar{k})$, denoted $h_{D}(P)$. In fact, up to a bounded function, $h_{D}$ depends only on the linear equivalence class of $D$. For appropriately chosen local Weil functions $\lambda_{D, v}, v \in M_{k}$, we can decompose the height into a sum of local Weil functions:

$$
h_{D}(P)=\sum_{v \in M_{k}} \lambda_{D, v}(P)
$$

for $P$ in $X(k) \backslash \operatorname{Supp} D$. We refer the reader to [2], [5], and [22] for a more thorough discussion of the definitions and properties of local Weil functions and height functions.

For a point $P$ in $X(\bar{k})$, we define the absolute logarithmic discriminant by

$$
d(P)=\frac{1}{[k(P): \mathbb{Q}]} \log \left|D_{k(P) / \mathbb{Q}}\right|,
$$

where $D_{k(P) / \mathbb{Q}}$ is the discriminant of $k(P)$ over $\mathbb{Q}$. As usual, we let $\mathcal{O}_{X}$ denote the structure sheaf of $X$ and we let $K_{X}$ denote a canonical divisor of $X$. We say that $D$ is a big divisor on $X$ if

$$
\limsup _{m \rightarrow \infty} \frac{h^{0}\left(X, \mathcal{O}_{X}(m D)\right)}{m^{\operatorname{dim} X}}>0
$$


In particular, an ample divisor is big. More generally, a well-known lemma of Kodaira [6, Addendum] gives that on a projective variety a divisor is big if and only if some positive multiple of the divisor can be written as the sum of an ample divisor and an effective divisor.

We say that an effective divisor $D$ is a normal crossings divisor (or has normal crossings) if every point $P \in \operatorname{Supp} D$ has an analytic open neighborhood in $X(\mathbb{C})$ with analytic local coordinates $z_{1}, \ldots, z_{\operatorname{dim} X}$ such that $D$ is locally defined by $z_{1} \cdot z_{2} \cdots z_{i}=0$ for some $i$. In particular, a normal crossings divisor is a reduced effective divisor.

\section{The Dependence of the exceptional set on $A$}

We now show that the exceptional set must be allowed to depend on the divisor $A$.

Theorem 3.1. The exceptional set $Z=Z(r, \epsilon, X, D, A)$ cannot be chosen independently of $A$, even for $r=1, D=0$, and varying only over ample divisors $A$.

As mentioned earlier, this theorem is very closely related to the fact that there are examples where the exceptional set $Z(r, \epsilon, X, D, A)$ must depend on $\epsilon$.

Proof. Let $X$ be a nonsingular projective surface over a number field $k$ with infinitely many distinct curves $C \cong \mathbb{P}_{k}^{1}$ having intersection number $K_{X} \cdot C>0$. An example of such a surface was explicitly given in [11, Ex. 2.4]. Let $0<\epsilon<\frac{1}{2}$. It suffices to show that for any such curve $C$, there exists an ample divisor $A$ with $C \subset Z(r=1, \epsilon, X, D=0, A)$. Let $C$ be such a curve. We first claim that there exists an ample divisor $A^{\prime}$ on $X$ satisfying $\operatorname{gcd}\left(A^{\prime} . C, C . C\right) \leq 2$. Note that since $C \cong \mathbb{P}^{1}$, by the adjunction formula, $K_{X} . C=-2-C . C$ and $C . C<0$. It follows that for any ample divisor $A^{\prime \prime}$ on $X$, either $A^{\prime}=A^{\prime \prime}$ or $A^{\prime}=n A^{\prime \prime}+K_{X}$, for some sufficiently large integer $n$, will satisfy $A^{\prime}$ is ample and $\operatorname{gcd}\left(A^{\prime} . C, C . C\right) \leq 2$. Since $A^{\prime} . C>0$ and $C . C<0$, we may choose positive integers $i$ and $j$ such that $i\left(A^{\prime} . C\right)+j(C . C)=\operatorname{gcd}\left(A^{\prime} . C, C . C\right) \leq 2$. Let $A=i A^{\prime}+j C$. We claim that $A$ is ample. By construction, we have $A . C>0$. For any curve $C^{\prime} \neq C, C . C^{\prime} \geq 0$ and $A^{\prime} . C^{\prime}>0$, so $A . C^{\prime}>0$. Moreover, since $A^{\prime} . A>0$ and $C . A>0$, we have $A^{2}>0$. It follows from the Nakai-Moishezon criterion [4, p. 365] that $A$ is ample. Since $K_{X} . C-\epsilon A . C \geq 1-2 \epsilon>0$, infinitely many points $P$ in $C(k)$ will satisfy $h_{K}(P)>\epsilon h_{A}(P)$. So we must have $C \subset Z(r=1, \epsilon, X, D=0, A)$, as was to be shown.

\section{ELLIPTIC SURFACES}

\section{AND THE DEPENDENCE OF THE EXCEPTIONAL SET ON $r$}

In this section we will study certain instances of Vojta's Conjecture on elliptic surfaces. We assume throughout that our (nonsingular, projective) elliptic surfaces are nonconstant and relatively minimal (i.e., no fiber contains an exceptional curve of the first kind). Recall that such an elliptic surface is called semistable if every singular fiber has Kodaira type $I_{n}$ for some $n \geq 1$. In particular, on a semistable elliptic surface $X$, every fiber is a normal crossings divisor.

We will find it convenient to consider a modified version of Vojta's Conjecture where the coefficient of $d(P)$ is a function of the dimension of $X$. Let $f: \mathbb{N} \rightarrow \mathbb{R}$ be a fixed positive real-valued function. 
Conjecture 4.1 (Modified Vojta Conjecture with function $f(n)$ ). Let $X$ be $a$ nonsingular complete variety of dimension $n$ with canonical divisor $K$. Let $D$ be a normal crossings divisor on $X$, and let $k$ be a number field over which $X$ and $D$ are defined. Let $S$ be a finite set of places of $k, A$ a big divisor on $X, \epsilon>0$, and $r$ a positive integer. Then there exists a proper Zariski closed subset $Z=$ $Z(f, r, \epsilon, X, D, A)$ of $X$ such that

$$
m_{D, S}(P)+h_{K}(P) \leq f(n) d(P)+\epsilon h_{A}(P)+O(1)
$$

for all points $P \in X(\bar{k}) \backslash Z$ with $[k(P): k] \leq r$.

We now prove the main result of this section.

Theorem 4.2. Let $X$ be a semistable elliptic surface over $\mathbb{P}^{1}$ with a section over a number field $k$. Let $D$ be a sum of an even number $N$ of fibers, containing the singular fibers. If Conjecture 4.1 holds for $X, D$, and a function $f$ satisfying $f(2)<1+\frac{\chi\left(\mathcal{O}_{X}\right)}{N-2}$, then the exceptional set $Z(f, r, \epsilon, X, D, A)$ must depend on the parameter $r$.

Proof. By enlarging $k$, we may assume that every fiber in $D$ is defined over $k$ and that the group of units $\mathcal{O}_{k}^{*}$ is infinite. Let $\pi: X \rightarrow \mathbb{P}^{1}$ be the fibration and let $\sigma: \mathbb{P}^{1} \rightarrow X$ be a section over $k$ (which we also identify with its image). We first claim that $\sigma \backslash D \cong \mathbb{P}^{1} \backslash\{N$ points $\}$ contains an infinite set of integral points $T$ with $[k(P): k] \leq \frac{N}{2}$ for all $P \in T$. Let $R=\sigma \cap D=R_{1} \cup R_{2}$, where $R_{1}$ and $R_{2}$ are disjoint sets each with $\frac{N}{2}$ elements of $R$. Let $\psi$ be a rational function over $k$ on $\mathbb{P}^{1}$ having zeros exactly at points in $R_{1}$ and poles exactly at points in $R_{2}$. Then $\psi$ defines a morphism $\psi: \sigma \backslash D \rightarrow \mathbb{G}_{m}$ of degree $\frac{N}{2}$. Since $\mathbb{G}_{m}$ contains an infinite set $T^{\prime}$ of integral points over $k$ ( $\mathcal{O}_{k}^{*}$ was assumed infinite), the set $T=\psi^{-1}\left(T^{\prime}\right)$ gives an infinite set of integral points on $\sigma \backslash D$ with $[k(P): k] \leq \frac{N}{2}$ for all $P \in T$.

Taking $\sigma$ to be the zero section on $X$, we have a rational map $[m]: X \rightarrow X$ induced by the fiberwise multiplication-by- $m$ map on the nonsingular fibers of $X$. Let $C \neq \sigma$ be an irreducible component of the Zariski closure of $[m]^{-1}(\sigma \backslash D)$. Let $L \supset k$ be a number field that is a field of definition for $C$. Then $[m]$ induces a morphism (over $L$ ) $\phi: C \rightarrow \sigma$. Let $\tilde{T}=\phi^{-1}(T)$. Let $A=\sigma+F$, where $F$ is some fiber. Then $A$ is a big divisor on $X$. Indeed, from the intersection numbers $\sigma . F=1$ and $F^{2}=F . F=0$, it is immediate that for some positive integer $n$ we have $(\sigma+n F)^{2}>0$. Using Riemann-Roch (e.g., [4, pp. 364-365]), this implies that $\sigma+n F$ is big, which in turn implies that $n(\sigma+F)=(n-1) \sigma+(\sigma+n F)$ is big. Finally, since a positive multiple of $\sigma+F$ is big, it follows from the definition that $\sigma+F$ is itself big, as desired. Now let $S$ be the set of Archimedean places of $k$. We claim that for any $\epsilon>0$ and any constant $c$, all but finitely many points $P$ in $\tilde{T}$ satisfy

$$
m_{D, S}(P)+h_{K}(P)>\left(1+\frac{\chi\left(\mathcal{O}_{X}\right)-2 \epsilon}{N-2}\right) d(P)+\epsilon h_{A}(P)+c .
$$

Let us first show that this claim implies the theorem. Every point $P$ in $\tilde{T}$ satisfies $[k(P): k] \leq \frac{N}{2}[L: k] \operatorname{deg} \phi$. Since $f(2)<1+\frac{\chi\left(\mathcal{O}_{X}\right)}{N-2}$, it follows from (4.2) that for sufficiently small $\epsilon$ (depending only on $f(2)), C \subset Z(f, r, \epsilon, X, D, A)$, where $r=r(C)=\frac{N}{2}[L: k] \operatorname{deg} \phi$. As we vary the integer $m$, we obtain infinitely many distinct such curves $C$ (with different values $r=r(C)$ ), obtaining the desired result. 
We now show (4.2). First, since $\tilde{T}=\phi^{-1}(T)$ and $T$ is a set of integral points on $\sigma \backslash D$, it follows that $\tilde{T}$ is a set of integral points on $C \backslash D$. Thus,

$$
m_{D, S}(P)=h_{D}(P)+O(1), \quad \forall P \in \tilde{T} .
$$

The canonical divisor is given by $K_{X}=\left(\chi\left(\mathcal{O}_{X}\right)-2\right) F$ [7, Th. 12], where $F$ is a fiber. We have $h_{A}=h_{\sigma}+h_{F}+O(1)$. It is known that $\sigma \cap C=\emptyset$ (see, e.g., 14, p. 72, Prop. (VII.3.2)]). It follows that $h_{\sigma}$ is bounded on $C$. So

$$
h_{A}(P)=h_{F}(P)+O(1), \quad \forall P \in C .
$$

Since $D \sim N F$, it follows that for all $P$ in $\tilde{T}$ we have

$$
m_{D, S}(P)+h_{K}(P)-\epsilon h_{A}(P)=\left(N+\chi\left(\mathcal{O}_{X}\right)-2-\epsilon\right) h_{F}(P)+O(1) .
$$

Note that $\left.F\right|_{C}$ is ample, and so for any infinite set of points of bounded degree on $C$ we have $h_{F}(P) \rightarrow \infty$. Thus, for any constant $c$, all but finitely many points $P$ in $\tilde{T}$ satisfy

$$
m_{D, S}(P)+h_{K}(P)-\epsilon h_{A}(P)>\left(N+\chi\left(\mathcal{O}_{X}\right)-2-2 \epsilon\right) h_{F}(P)+c .
$$

Comparing with (4.2), to finish the proof it suffices to show that

$$
d(P) \leq(N-2) h_{F}(P)+O(1), \quad \forall P \in \tilde{T} .
$$

This will follow from the following two results.

Theorem 4.3 (Mahler [12]). The inequality

$$
d(P) \leq(2 r-2) h(P)+O(1)
$$

holds for all $P \in \mathbb{P}^{1}(\bar{k})$ with $[k(P): k] \leq r$.

Theorem 4.4 (Chevalley-Weil). Let $\phi: X \rightarrow Y$ be a finite morphism of nonsingular projective varieties, with ramification divisor $R$ on $X$. Let $T$ be a set of integral points of bounded degree on $X \backslash R$. Then

$$
d(P) \leq d(\phi(P))+O(1)
$$

for all $P$ in $T$.

For a more general quantitative version of the Chevalley-Weil theorem, see 22, Th. 5.1.6].

Since $[k(P): k] \leq \frac{N}{2}$ for points $P$ in $T$, by Theorem 4.3 we have

$$
d(P) \leq(N-2) h(P)+O(1), \quad \forall P \in T .
$$

The map $\phi: C \rightarrow \sigma$ is unramified outside of $\left.D\right|_{C}$. So by Theorem 4.4

$$
d(Q) \leq d(\phi(Q))+O(1), \quad \forall Q \in \tilde{T} .
$$

By functoriality, $h_{F}(P)=h(\pi(P))+O(1)$ for all $P \in X(\bar{k})$. So

$$
h_{F}(Q)=h_{F}(\phi(Q))+O(1)=h(\phi(Q))+O(1), \quad \forall Q \in C(\bar{k}) .
$$

Then combining (4.4), (4.5), and (4.6), we obtain (4.3) as desired.

To optimally apply Theorem 4.2, we want to minimize the number of singular fibers on a semistable elliptic surface relative to $\chi\left(\mathcal{O}_{X}\right)$. This problem was studied by Shioda in 17 . 
Theorem 4.5 (Shioda). Let $X$ be a semistable elliptic surface over $\mathbb{P}^{1}$ with a section. Let $N$ be the number of singular fibers. Then $N \geq 2 \chi\left(\mathcal{O}_{X}\right)+2$. Furthermore, for any positive integer $\chi$, there exists such an $X$ with a section over a number field $k, \chi\left(\mathcal{O}_{X}\right)=\chi$, and exactly $N=2 \chi+2$ singular fibers.

The construction of semistable elliptic surfaces with a minimal number of singular fibers is related to so-called Davenport-Stothers triples and to polynomials which achieve equality in the polynomial $a b c$ theorem. As a consequence of Shioda's result, we obtain:

Corollary 4.6. If Conjecture 4.1 holds for $r=\infty$, then we must have $f(2) \geq \frac{3}{2}$.

A natural question to ask is whether Theorem 4.2 or Corollary 4.6 can be improved by looking at elliptic surfaces $X$ (with a section) over a higher genus base curve $B$. In general, for such an $X$ and a base curve $B$, we have Kodaira's formula [7, Th. 12]

$$
K_{X}=\pi^{*}\left(K_{B}+E\right),
$$

where $E$ is some divisor of degree $\chi\left(\mathcal{O}_{X}\right)$ on $B$, and an inequality of Song and Tucker [18, Eq. (2.0.3)]

$$
d(P) \leq(2 r+\epsilon) h_{\mathrm{pt}}(P)+h_{K_{B}}(P)+O(1)
$$

for all $P$ in $B(\bar{k})$ with $[k(P): k] \leq r$, where pt is any point of $B(k)$. Mimicking the proof of Theorem 4.2, we find that the key quantity is the ratio

$$
\frac{N+2 g-2+\chi\left(\mathcal{O}_{X}\right)}{N+2 g-2}=1+\frac{\chi\left(\mathcal{O}_{X}\right)}{N+2 g-2},
$$

where $N$ is the smallest even integer greater than or equal to the number of singular fibers of $X$. Unfortunately, we have the general inequality

$$
N \geq 2 \chi\left(\mathcal{O}_{X}\right)+2-2 g .
$$

Thus, it does not appear that one can improve on the constant $\frac{3}{2}$ in Corollary 4.6 by using a base curve of higher genus. More generally, in the case $X$ is not necessarily semistable, we have the inequality [16, Cor. 2.7]

$$
\mu+2 \alpha \geq 2 \chi\left(\mathcal{O}_{X}\right)+2-2 g,
$$

where $\mu$ and $\alpha$ are the number of multiplicative and additive singular fibers, respectively. This gives some hope of using additive singular fibers to improve the number $\frac{3}{2}$ in Corollary 4.6. In this case, however, the singular fibers are no longer normal crossings divisors (as is required in Vojta's Conjecture), and this appears to prevent one, at least with our method, from improving on Corollary 4.6.

Using a trick of Vojta [22, Prop. 5.4.1], we can use our results on elliptic surfaces to construct varieties $X$ such that $D=0$ and the exceptional set $Z(r, \epsilon, X, D, A)$ must depend on the parameter $r$.

Theorem 4.7. There exist a nonsingular projective surface $V$ defined over a number field $k$; curves $C_{i} \subset V, i \in \mathbb{N}$, with $\bigcup_{i} C_{i}$ Zariski-dense in $V$; a big divisor $A$; and a function $g: \mathbb{N} \rightarrow \mathbb{N}$ such that for any $i \in \mathbb{N}, \delta>0$, sufficiently small $\epsilon>0$, and constant $c_{i}$,

$$
h_{K_{V}}(P)>\left(\frac{3}{2}-\delta\right) d(P)+\epsilon h_{A}(P)+c_{i}
$$


for an infinite set of points $P \in C_{i}$ with $[k(P): k] \leq g(i)$. In particular, for any set of constants $c_{i}, i \in \mathbb{N}, \delta>0$, and any sufficiently small $\epsilon>0$, the inequality

$$
h_{K_{V}}(P)>\left(\frac{3}{2}-\delta\right) d(P)+\epsilon h_{A}(P)+c_{[k(P): k]}
$$

holds for a Zariski-dense set of points $P \in V(\bar{k})$.

Proof. By Theorem 4.5, there exists a semistable elliptic surface $X$ over $\mathbb{P}^{1}$ with a section over a number field $k$ and $N=2 \chi\left(\mathcal{O}_{X}\right)+2$ singular fibers. Let $D$ be the sum of the singular fibers, which we may assume are all defined over $k$. By Theorem 4.2 and its proof, there exist infinitely many distinct curves $C_{i}^{\prime} \subset X$, $i \in \mathbb{N}$, with an infinite set of $D$-integral points $T_{i}$ of bounded degree, and an ample divisor $A^{\prime}$, such that for any constant $c_{i}$ and sufficiently small $\epsilon>0$,

$$
m_{D, S}(P)+h_{K_{X}}(P)>\left(\frac{3}{2}-\delta\right) d(P)+\epsilon h_{A^{\prime}}(P)+c_{i}
$$

for all but finitely many points $P$ in $T_{i}$.

We may assume one of the singular fibers lies over the point at infinity of $\mathbb{P}^{1}$. Let $t_{1}, \ldots, t_{N-1} \in k$ be the projections of the other singular fibers. Let $M$ be a large enough positive integer such that

$$
m_{D, S}(P)<\frac{M \epsilon}{2} h_{A^{\prime}}(P)+O(1)
$$

for all $P$ in $X(\bar{k}) \backslash D$. Let $B$ be the nonsingular projective curve associated to $k\left(\sqrt[M]{t-t_{1}}, \ldots, \sqrt[M]{t-t_{N-1}}\right)$. We have a natural morphism $\phi: B \rightarrow \mathbb{P}^{1}$ of degree $M^{N-1}$, with ramification index $M$ at each point above $t_{1}, \ldots, t_{N-1}, \infty$ and unramified everywhere else. Let $V=X \times_{\mathbb{P}^{1}} B$ and let $\pi: V \rightarrow X$ be the projection map. From the above, it follows easily that the ramification divisor $R$ of $\pi$ is given by $R=\frac{M-1}{M} \pi^{*} D$. We also have the formula $K_{V}=\pi^{*} K_{X}+R$. Since the set of points $T_{i}$ is $D$-integral, by Theorem 4.4 we have

$$
d(Q) \leq d(\pi(Q))+O(1)
$$

for all points $Q$ in $\pi^{-1}\left(T_{i}\right)$. Let $A=\pi^{*} A^{\prime}$, a big divisor. Then for all $Q \in \pi^{-1}\left(T_{i}\right)$, setting $P=\pi(Q)$, we have (up to $O(1)$ )

$$
\begin{aligned}
h_{K_{V}}(Q) & =h_{\pi^{*} K_{X}}(Q)+h_{R}(Q)=h_{K_{X}}(P)+\frac{M-1}{M} h_{D}(P) \\
& \geq h_{K_{X}}(P)+\frac{M-1}{M} m_{D, S}(P) \\
& \geq h_{K_{X}}(P)+m_{D, S}(P)-\frac{\epsilon}{2} h_{A^{\prime}}(P) \\
& \geq\left(\frac{3}{2}-\delta\right) d(P)+\frac{\epsilon}{2} h_{A^{\prime}}(P) \\
& \geq\left(\frac{3}{2}-\delta\right) d(Q)+\frac{\epsilon}{2} h_{A}(Q) .
\end{aligned}
$$

This yields the theorem, taking $C_{i}$ to be an appropriate curve in $\pi^{-1}\left(C_{i}^{\prime}\right)$ for each $i \in \mathbb{N}$. 
Finally, let us discuss some recent results in a related setting that are closely connected to the results of this section. In a geometric setting, Autissier, ChambertLoir, and Gasbarri have recently shown [1]:

Theorem 4.8 (Autissier, Chambert-Loir, Gasbarri). Let $n \geq 2$ be an integer and $k$ an algebraically closed field of characteristic zero. There exists a nonsingular projective variety $X$ over $k$ of dimension $n$, with canonical divisor $K$, and projective curves $C_{i} \subset X, i \in \mathbb{N}$, such that the following are true:

(1) $\bigcup_{i \in \mathbb{N}} C_{i}$ is Zariski-dense in $X$.

(2) Let $A$ be an ample divisor on $X$ and let $\delta>0$. For all sufficiently small $\epsilon>0$,

$$
C_{i} . K>(n-\delta) \chi\left(C_{i}\right)+\epsilon C_{i} . A,
$$

for all but finitely many $i \in \mathbb{N}$.

Here, for a projective curve $C$, we let $\chi(C)=2 g(C)-2$ where $g(C)$ is the geometric genus of $C$. The terms $C . K, C . A$, and $\chi(C)$ are analogous to (the unnormalized versions of) $h_{K}(P), h_{A}(P)$, and $d(P)$, respectively, in Conjecture 1.1 As noted in [1, the gonalities of the curves $C_{i}$ constructed in [1 in Theorem 4.8 go to infinity with $i$ (the gonality is an analogue of the degree $[k(P): k]$ ). Theorem 4.8 shows that in a geometric setting the analogue of inequality (1.1) in Vojta's Conjecture does not hold uniformly without at least a $\operatorname{dim} X$ factor in front of (the analogue of) the $d(P)$ term.

On the other hand, for nonsingular curves on minimal surfaces of general type, Miyaoka [15] proved:

Theorem 4.9 (Miyaoka). Let $X$ be a nonsingular minimal complex projective surface of general type with canonical divisor $K$. Let $A$ be an ample divisor on $X$ and let $\epsilon>0$. Then

$$
C . K \leq \frac{3}{2} \chi(C)+\epsilon C . A+O(1)
$$

for all nonsingular projective curves $C$ in $X$.

It is unclear if the appearance of the coefficient $\frac{3}{2}$ in both Miyaoka's result and the results given here is coincidence or holds some deeper significance.

\section{ACKNOWLEDGMENTS}

The author thanks Antoine Chambert-Loir for many fruitful discussions and for introducing him to and explaining his joint paper 1 with Autissier and Gasbarri (which was an inspiration for the present paper). The author thanks William Cherry for helpful comments on an earlier draft and, in particular, for bringing to his attention the paper 19. He thanks Tom Tucker and Paul Vojta for further useful discussions. He thanks the anonymous referee for several suggestions that certainly improved the quality of the paper. He thanks the Institute for Advanced Study for providing a nice research environment in which some of this research was done.

\section{REFERENCES}

1. P. Autissier, A. Chambert-Loir, and C. Gasbarri, On the canonical degrees of curves in varieties of general type, arXiv:1003.3804v1 [math.AG] (preprint).

2. E. Bombieri and W. Gubler, Heights in Diophantine geometry, New Mathematical Monographs, vol. 4, Cambridge University Press, Cambridge, 2006. MR 2216774 (2007a:11092) 
3. W. Cherry, The Nevanlinna error term for coverings, generically surjective case, Proceedings Symposium on Value Distribution Theory in Several Complex Variables (Notre Dame, IN, 1990), Notre Dame Math. Lectures, vol. 12, Univ. Notre Dame Press, 1992, pp. 37-53. MR.1243017 (95b:30040)

4. R. Hartshorne, Algebraic geometry, Graduate Texts in Mathematics, No. 52, Springer-Verlag, New York, 1977. MR0463157 (57:3116)

5. M. Hindry and J. H. Silverman, Diophantine geometry. An introduction, Graduate Texts in Mathematics, vol. 201, Springer-Verlag, New York, 2000. MR1745599 (2001e:11058)

6. S. Kobayashi and T. Ochiai, Mappings into compact complex manifolds with negative first Chern class, J. Math. Soc. Japan 23 (1971), 137-148. MR0288316 (44:5514)

7. K. Kodaira, On the structure of compact complex analytic surfaces. I, Amer. J. Math. 86 (1964), 751-798. MR0187255 (32:4708)

8. S. Lang, The error term in Nevanlinna theory. II, Bull. Amer. Math. Soc. (N.S.) 22 (1990), no. 1, 115-125. MR1003864 (90k:32080)

9. - Number theory. III. Diophantine geometry, Encyclopaedia of Mathematical Sciences, vol. 60, Springer-Verlag, Berlin, 1991. MR.1112552 (93a:11048)

10. S. Lang and W. Cherry, Topics in Nevanlinna theory, Lecture Notes in Mathematics, vol. 1433, Springer-Verlag, Berlin, 1990, with an appendix by Zhuan Ye. MR1069755 (91k:32025)

11. A. Levin, D. McKinnon, and J. Winkelmann, On the error terms and exceptional sets in conjectural second main theorems, Q. J. Math. 59 (2008), no. 4, 487-498. MR2461270 (2010f:11127)

12. K. Mahler, An inequality for the discriminant of a polynomial, Michigan Math. J. 11 (1964), 257-262. MR0166188 (29:3465)

13. M. McQuillan, Old and new techniques in function field arithmetic, preprint.

14. R. Miranda, The basic theory of elliptic surfaces, Dottorato di Ricerca in Matematica [Doctorate in Mathematical Research], ETS Editrice, Pisa, 1989. MR.1078016 (92e:14032)

15. Y. Miyaoka, The orbibundle Miyaoka-Yau-Sakai inequality and an effective BogomolovMcQuillan theorem, Publ. Res. Inst. Math. Sci. 44 (2008), no. 2, 403-417. MR2426352 (2009g:14043)

16. T. Shioda, On elliptic modular surfaces, J. Math. Soc. Japan 24 (1972), 20-59. MR0429918 $(55: 2927)$

17. The abc-theorem, Davenport's inequality and elliptic surfaces, Proc. Japan Acad. Ser. A Math. Sci. 84 (2008), no. 4, 51-56. MR2409500 (2009g:14040)

18. X. Song and T. Tucker, Dirichlet's theorem, Vojta's inequality, and Vojta's conjecture, Compositio Math. 116 (1999), no. 2, 219-238. MR1686848 (2000d:11085)

19. C. L. Stewart and R. Tijdeman, On the Oesterlé-Masser conjecture, Monatsh. Math. 102 (1986), no. 3, 251-257. MR863221 (87k:11077)

20. M. van Frankenhuijsen, A lower bound in the abc conjecture, J. Number Theory 82 (2000), no. 1, 91-95. MR.1755155 (2001m:11109)

21. _ 127 (2007), no. 2, 292-300. MR2362438 (2008j:11087)

22. P. Vojta, Diophantine approximations and value distribution theory, Lecture Notes in Mathematics, vol. 1239, Springer-Verlag, Berlin, 1987. MR883451 (91k:11049)

23. _ A refinement of Schmidt's subspace theorem, Amer. J. Math. 111 (1989), no. 3, 489-518. MR.1002010 (90f:11054)

24. _ A more general abc conjecture, Internat. Math. Res. Notices (1998), no. 21, 11031116. MR:1663215 (99k:11096)

25. K. Yamanoi, The second main theorem for small functions and related problems, Acta Math. 192 (2004), no. 2, 225-294. MR2096455 (2006g:30050)

Department of Mathematics, Michigan State University, East Lansing, Michigan 48824

E-mail address: adlevin@math.msu.edu 